University of Louisville

ThinkIR: The University of Louisville's Institutional Repository

Faculty Scholarship

$1-2015$

\title{
Do depressive symptoms predict the incidence of myocardial infarction independent of hopelessness?
}

\author{
Patrick Pössel \\ University of Louisville \\ Amanda M. Mitchell \\ University of Louisville \\ Kimmo Ronkainen \\ University of Eastern Finland \\ George A. Kaplan \\ University of Michigan-Ann Arbor \\ Jussi Kauhanen \\ University of Eastern Finland \\ See next page for additional authors
}

Follow this and additional works at: https://ir.library.louisville.edu/faculty

Part of the Counseling Psychology Commons, and the Health Psychology Commons

\section{Original Publication Information \\ Pössel, Patrick, Amanda M. Mitchell, Kimmo Ronkainen, George A. Kaplan, Jussi Kauhanen and Maarit Valtonen. "Do Depressive Symptoms Predict the Incidence of Myocardial Infarction Independent of Hopelessness?" 2015. Journal of Health Psychology 20(1): 60-68. \\ https://doi.org/10.1177/1359105313498109}

This Article is brought to you for free and open access by ThinkIR: The University of Louisville's Institutional Repository. It has been accepted for inclusion in Faculty Scholarship by an authorized administrator of ThinkIR: The University of Louisville's Institutional Repository. For more information, please contact thinkir@louisville.edu. 


\section{Authors}

Patrick Pössel, Amanda M. Mitchell, Kimmo Ronkainen, George A. Kaplan, Jussi Kauhanen, and Maarit Valtonen

This article is available at ThinkIR: The University of Louisville's Institutional Repository: https://ir.library.louisville.edu/ 
Depressive Symptoms, Hopelessness, and MI incidence

Do Depressive Symptoms Predict the Incidence of Myocardial Infarction Independent of Hopelessness?

Patrick Pössel, Amanda M. Mitchell, Department of Educational and Counseling Psychology, University of Louisville, Louisville, KY, USA

Kimmo Ronkainen

Intitute of Public Health and Clinical Nutrition, University of Eastern Finland, Kuopio, Finland George A. Kaplan

Center for Social Epidemiology and Population Health, University of Michigan, Ann Arbor, Michigan, USA

Jussi Kauhanen

Institute of Public Health and Clinical Nutrition, University of Eastern Finland, Kuopio, Finland Maarit Valtonen

Institute of Public Health and Clinical Nutrition, University of Eastern Finland, Kuopio, Finland

Corresponding author:

Patrick Pössel, Dr. rer. soc.

Dep. of Educational and Counseling Psychology

University of Louisville

2301 S. Third Street

Louisville, KY 40292 
Depressive Symptoms, Hopelessness, and MI incidence

USA

$+1-(502) 852-0623$ (office)

$+1-(502) 852-0629$ (fax)

e-mail: patrick.possel@louisville.edu 
Depressive Symptoms, Hopelessness, and MI incidence

\begin{abstract}
Depression and hopelessness predict myocardial infarction (MI), but it is unclear whether depression and hopelessness are independent predictors of MI incidents. Hopelessness, depression, and MI incidence rate 18 years later were measured in 2005 men. Cox regressions were conducted with hopelessness and depression serving as individual predictors of MI. Another Cox model examined whether the two predictors predict MI when adjusting for each other. Depression and hopelessness predicted MI in independent regressions but when adjusting for each other, hopelessness, but not depression, predicted MI incidents. Thus, these results suggest that depression and hopelessness are not independent predictors of MI.
\end{abstract}

Keywords: population-based; longitudinal follow-up study; hopelessness; depressive symptoms; myocardial infarction 
The Global Burden of Disease study launched by the World Health Organization predicts coronary heart disease (CHD) (incl. myocardial infarction; MI) and depression will be two of the three most disabling disorders worldwide by 2030, only surpassed by HIV/AIDS (Mathers and Loncar, 2006). In high-income countries, including the USA, depression and CHD will be the two most disabling disorders with $15.7 \%$ of total disability-adjusted life years (DALYS).

Beyond this, CHD is the number one cause of death globally and in high-income countries and is projected to remain the leading cause of death with $13.4 \%$ and $15.8 \%$ of total deaths, respectively (Mathers and Loncar, 2006). Currently, an estimated 83.6 million or 1/3 of all American adults have one or more types of CHD and this number is projected to increase to 40.8\% by the year 2030 (American Heart Association, 2013). Additionally, CHD was the underlying cause of death in $32.3 \%$ of all deaths in 2009 ; it is the leading cause of death for both males and females (American Heart Association, 2013).

Several empirical studies have demonstrated that depressive symptoms are prospectively associated with incidence of CHD (Leung et al., 2012; Nicholson et al., 2006; Van der Kooy et al., 2007), even after adjusting for other risk factors. For example, the Canadian National Population Health Survey revealed that after adjusting for gender and age, depressive symptoms increase the risk for hypertension and heart disease two years later (Patten et al., 2008). Further recent research indicates that the association between depressive symptoms and future CHD incidents is particularly strong in men (Fiedorowicz et al., 2011). Finally, a systematic review identified 22 longitudinal studies researching depressive symptoms as a risk factor for the development of CHD (Kuper et al., 2002). In this review, 15 of the identified studies supported a link between depressive symptoms and CHD, while only 7 could not provide a clear link. 
Increased knowledge about the relationship between depressive symptoms and CHD opens possibilities for new ways of attenuating the influence of depressive symptoms on CHD (Grippo and Johnson, 2002). Consistent with this consideration, recent literature reflects an interest in identifying which specific aspects of depression are strong predictors of CHD. For example, particular cognitive aspects of depressive symptoms (incl. pessimism, past failure, selfcriticalness, worthlessness) were associated with cardiac morbidity and mortality in a study with coronary artery bypass graft patients (Tully et al., 2011). Another cognitive aspect of depression is hopelessness- the negative view of the future. Hopelessness is characterized as a "toxic" symptom of depression and it often occurs with severe episodes of depression (Brown and Harris, 1978). Nevertheless, hopelessness is not recognized as a symptom of depression in the DSM-IV-TR (American Psychiatric Association, 2000) and two prominent models explaining the development and maintenance of depression identify hopelessness as a risk factor and not as a symptom of depression (Abramson et al., 1989; Beck, 1976). Thus, hopelessness may be distinct from depression in its associations with CHD.

This hypothesis is supported by multiple studies. For example, a 12-year longitudinal study with 45 to 77 year-old adults without a history of CHD or other serious illness at baseline revealed that the relative risk of fatal and nonfatal CHD increased by $50 \%$ and $40 \%$, respectively, for each increase in level of hopelessness after adjusting for age, gender, ethnicity, marital status, smoking, total cholesterol, systolic blood pressure, body mass index, alcohol use, and physical activity (Anda et al., 1993). Furthermore, moderate levels of hopelessness increased the relative risk of fatal and nonfatal CHD by approximately $50 \%$ more than depressive symptoms. Everson et al. (1996) revealed in their population-based longitudinal study that for men with no known history of angina pectoris or MI, those reporting high levels of 
hopelessness were at a significantly increased risk for a first MI, relative to men scoring low on the measure for hopelessness. Men with moderate levels of hopelessness showed a $20 \%$ to $30 \%$ increased risk of MI, relative to men with low hopelessness scores, but these elevations were not significant. While Everson et al. (1996) tested the association between hopelessness and CHD incidents, after adjusting for depressive symptoms, the relationship between depressive symptoms and CHD has not been studied while adjusting for hopelessness.

The cross-sectional Study of Women's Health Across the Nation Heart Study (SWAN; Whipple et al., 2009) with healthy women found that hopelessness significantly predicted mean and maximum carotid artery intima-media thickening (IMT) while depressive symptoms predicted IMT only marginally, both while adjusting for age, race/ethnicity (i.e., European American and African-American), income, BMI, SBP, and smoking. Moreover, after entering both hopelessness and depressive symptoms in the same model and continuing to adjust for all other variables, hopelessness but not depressive symptoms remained a significant predictor of IMT. Other publications have found that hopelessness predicts hypertension (Everson et al., 2000) and carotid atherosclerosis (Pollitt et al., 2005), both biological risk factors of CHD, even after adjusting for depressive symptoms. Given these findings, the question of whether hopelessness and depressive symptoms are longitudinally and independently associated with CHD becomes important to address.

Based on the above theoretical considerations (Abramson et al., 1989; Beck, 1976) and empirical findings (Anda et al., 1993; Everson et al., 1996; 2000; Pollitt et al., 2005; Whipple et al., 2009), in this study we predicted that hopelessness would be associated with depressive symptoms and MI incidents up to 18 years later. Further, we expected that the association between depressive symptoms and MI incidents would no longer be significant when adjusting 
for hopelessness, whereas the association between hopelessness and MI incidents were predicted not to be influenced by depressive symptoms.

\section{Methods}

\section{Participants}

This study utilized data collected as part of the Kuopio Ischemic Heart Disease (KIHD) study, a longitudinal population-based study examining risk factors of several serious health conditions (e.g., MI). Participants were enrolled in the study between March 1984 and December 1989, and the population consisted of 2682 men. Further details to recruitment and population are described in previous publications (Salonen, 1988). In the current analysis, participants were excluded from further analyses if they had experienced a CHD prior to baseline to remove the effectof CHD on depressive symptoms. Thus, 2005 men remained for examination, with an age range extending from 42 to 61 and a mean (SD) of 52.45 (5.32). All participating men gave their informed consent prior to their inclusion in the study, and the study followed the ethical standards of the Helsinki Declaration.

\section{Measures}

Hopelessness Scale (Everson et al., 1996). The hopelessness scale is comprised of two items assessing the degree to which an individual experiences feelings of hopelessness. The items were "I feel that it is impossible to reach the goals I would like to strive for" and "The future seems to me to be hopeless, and I can't believe that things are changing for the better." Participants responded to the items based on a 5-point Likert scale, ranging from 0 (absolutely agree) to 5 (absolutely disagree). To obtain a score on the hopelessness scale, the two items were reverse-coded and then summed. Hopelessness scores ranged from 0 to 8 with a mean 
Depressive Symptoms, Hopelessness, and MI incidence

(SD) of 2.51 (1.95). Internal consistency for the items with this particular population was $\alpha=$ .70.

\section{The Human Population Laboratory Depression Scale (HPLDS; Roberts and}

O'Keefe, 1981). The HPLDS is comprised of 18 items and assesses the degree to which an individual experiences depressive symptomatology. No items assessing hopelessness are included in the HPL. Participants responded to each item utilizing a true-false format, and depression scores were calculated by assigning a one-point value to each item that endorsed a depressive symptom and summing all of the items. Internal consistency for the items with this population was $\alpha=.71$. The HPLDS was developed to assess depressive symptoms in population-based samples and prior studies have utilized it to do such (Everson et al., 1998; Kaplan et al., 1987; Roberts et al., 1981; Tolmunen et al., 2004).

Myocardial Infarctions. Data on MI were obtained from the National Hospital Discharge Data Register by record linkage. The diagnostic classification of coronary events was based on symptoms, electrocardiographic findings, and cardiac enzyme elevations. Each suspected coronary event was coded according to the Ninth (code numbers 410-414; Centers for Disease Control and Prevention, 1998) or Tenth (code numbers I20-I25; World Health Organization, 2007) revision of the International Classification of Diseases (ICD) and was classified into (1) a definite MI, (2) a probable MI, (3) a typical acute chest pain episode of more than 20 min, indicating CHD, (4) an ischaemic cardiac arrest with successful resuscitation, or (5) no acute coronary event. The diagnosis was made by a physician using original patient records. All MI cases that occurred from the study entry until 31st December 2005 were included. Thus, average follow-up time was 15.2 years (range 12.3 to 18.0 years). In the present sample, 384 
men (19.2\%) experienced a MI between baseline and follow-up. If a participant had multiple events, the first event was considered as the end point.

\section{Data Analysis}

To test the hypotheses that hopelessness predicts both depressive symptoms and MI incidents and depressive symptoms is not an independent predictor of MI, one partial correlation and three different Cox proportional hazards regression models with MI as the dependent variable were calculated. First, a partial correlation between hopelessness and depressive symptoms was calculated. Second, two separate Cox models with hopelessness or depressive symptoms as predictors of MI incidents were calculated. Third, one Cox model with hopelessness and depressive symptoms as predictors of MI incidents was calculated. Finally, two paired t-tests were used to compare the regression weights in the Cox models with only one predictor (hopelessness or depressive symptoms) to the regression weights in the Cox models with both predictors (hopelessness and depressive symptoms). A significant t-test reveals that the regression between a predictor (e.g., depressive symptoms) and MI is substantially reduced by the introduction of the other predictor (e.g., hopelessness). In other words, the association between the first predictor (e.g., depressive symptoms) and MI is not independent from the second predictor (e.g., hopelessness; Haibe-Kains et al., 2008; Student, 1908).

The hypotheses would be correct when (a) hopelessness and depressive symptoms significantly correlate with each other; (b) both variables predict MI incidents when not adjusted for each other; (c) the regression weight of depressive symptoms, but not of hopelessness, is susbtantially reduced when comparing the regression weights in the Cox models with only one predictor (hopelessness or depressive symptoms) to the regression weights in the Cox models with both predictors (hopelessness and depressive symptoms; Haibe-Kains et al., 2008; Student, 
1908). All analyses (partial correlation and three Cox models) were adjusted for age, waist to hip ratio, low-density lipoprotein and high-density lipoprotein cholesterol levels, and resting systolic blood pressure.

\section{Results}

Depressive symptoms and hopelessness correlated with $r=.33(\mathrm{p}<.001)$ independent of adjustment for the influence of the control variables mentioned above. The adjusted Cox proportional hazards regression model with depressive symptoms as predictor of MI incidence revealed that higher depression scores were significantly associated with an increased risk for an MI ( $=.047$; Table 1). Moreover, the adjusted Cox model with hopelessness as predictor of MI incidence revealed that higher hopelessness scores were significantly associated with an increased risk for an MI ( $\mathrm{p}=.011$; Table 1). The adjusted Cox model that included both depressive symptoms and hopelessness as predictors of MI revealed that higher hopelessness scores $(\mathrm{p}=.047)$, but not depression scores $(\mathrm{p}=.212)$, remained significantly associated with an increased risk of an MI (Table 1).

Finally, paired t-tests comparing the regression weights in the Cox models with only one predictor (depressive symptoms or hopelessness) with the regression weights in the Cox models with both predictors (depressive symptoms and hopelessness) were calculated. These tests revealed that the regression weight of depression scores was significantly reduced when adjusting for hopelessness $(\mathrm{t}(2004)=2.18 ; \mathrm{p}=.029)$, while the regression weight of hopelessness did not change significantly when adjusting for depressive symptoms $(\mathrm{t}(2004)=$ $1.608 ; \mathrm{p}=.108)$

\section{Discussion}


The aim of this study was to test whether depressive symptoms and hopelessness are independent predictors of MI incidents (as one type of CHD). Consistent with the predictions, depressive symptoms and hopelessness correlated significantly with each other. Moreover, consistent with the hypothesis and previous empirical findings, depressive symptoms (Fiedorowicz et al., 2011; Nicholson et al., 2006; Patten et al., 2008; Van der Kooy et al., 2007) and hopelessness (Anda et al., 1993; Everson et al., 1996, 2000; Whipple et al., 2009) predicted MI incidents up to 18 years later when not adjusting for each other. Additionally, hopelessness remained a preditor of $\mathrm{MI}$ incidents, even after adjusting for depressive symptoms. Finally, the association between depressive symptoms at baseline and MI incidents was significantly reduced when adjusting for hopelessness, while the asssociation between baseline hopelessness and MI incidents did not change when adjusting for depressive symptoms. Summarized, the results are consistent with the hypothesis that hopelessness but not depressive symptoms is an independent predictor of MI.

Based on previous studies establishing depressive symptoms as risk factor for MI incidents (Fiedorowicz et al., 2011; Nicholson et al., 2006; Patten et al., 2008; Van der Kooy et al., 2007), multiple studies have attempted to reduce biological risk factors of MI incidents (e.g., Bot et al., 2011) or MI reoccurence itself (e.g., Glassman et al., 2009) by reducing depressive symptoms in patients with CHD. However, a recent meta-analysis of studies researching the effects of pharmacotherapeutic interventions to reduce depressive symptoms in patients with CHD did not improve the readmission and the mortality rate (Pizzi et al., 2011). Not all pharmaco- and psychotherapeutic interventions effective in treating depressive symptoms impact hopelessness equally well (see Pössel and Hautzinger, 2006 for a review). The finding of this study may provide one explanation for this particular finding in the meta-analysis. Thus, future 
studies of therapeutic approaches attempting to reduce MI incidents should study if a reduction of hopelessness as compared to a reduction of depressive symptoms improves MI incident rates. Although theoretical considerations (Abramson et al., 1989; Beck, 1976) led to the conclusion that hopelessness is a risk factor of both depressive symptoms and MI causing the impression of an association between depressive symptoms and MI incidents, it was not possible to test this hypothesis because depressive symptoms and hopelessness were measured in this study at the same time point. To be able to test the direction of the associations, a study with at least three waves of data collection and equal time lags between the waves is needed (Cole and Maxwell, 2003). This seems especially important given that some researchers conceptualize hopelessness as symptom of depression rather than a risk factor of depression (e.g., Do et al., 2010), despite that hopelessness is not listed as a symptom in the DSM-IV-TR (APA, 2000). Thus, an alternative interpretation of our findings might be that hopelessness is simply the "active factor" that connects depression to MI. Further possible explanations for our finding include but are not limited to (a) hopelessness may mediate the effect of depression on MI incidents, (b) a covariate may control for more variability in one predictor than in the other and thereby allow one predictor (e.g., hopelessness) to achieve greater predictive power relative to the other predictor (e.g., depressive symptoms), or (c) an omitted covariate (e.g., SES) is undercontrolled with respect to one predictor (e.g., hopelessness) but not another (e.g., depressive symptoms). Thus, the findings call not only for replication but also for additional explorations of the interplay of hopelessness, depressive symptoms, and other variables on hypertension, atherosclerosis, and heart rate variability as biological risk factors of MI incidents and other CHDs. 
Beyond that, while previous research has already studied the impact of some risk factors of depressive symptoms such as childhood abuse (Fuller-Thomson et al., 2010), perceived stress and perceived control (Cohen et al., 1999; Rosenbaum et al., 2012), a negative attribution style (Sanjuan et al., 2012), and low self-esteem (Stamatakis et al., 2004), many other risk factors of depressive symptoms have not yet been examined. Considering that both above mentioned psychological models of depression propose multiple cognitive risk factors of depression (Abramson et al., 1989; Beck, 1976) and that many of these risk factors of depression influence each other (e.g., Pössel, 2011; Pössel and Knopf, 2011), studies including multiple psychosocial factors seem especially fruitful. Further, multiple coping mechanisms like religious coping (Ai et al., 2013) and social support (Schmidt et al., 2012) are studied with cardiac or other severly ill patients. Thus, it would be important to explore the effects of these coping mechanisms on the relationship between depressive symptoms and CHD as well. Such studies will contribute to a further integration of psychological and biological models into a bio-psycho-social model of heart health.

Strengths of this study were that it included a large population-based design, an extended follow-up, and detailed assessments of different psychological factors and cardiovascular risk factors. However, hopelessness and depressive symptoms were measured using self-report questionnaires instead of structured interviews. In addition, the 2-item hopelessness scale that was used is fairly simple and has not been compared with other hopelessness scales. Nevertheless, it has been well-validated and in previous studies it has predicted various cardiovascular and metabolic outcomes (Do et al., 2010; Valtonen et al., 2008). Further, the cohort of this study included only middle-aged white men and the findings cannot be generalized to women or other ethnic groups. Given that men and women have been shown to report 
Depressive Symptoms, Hopelessness, and MI incidence

different levels of particular depressive symptoms, such as fatigue, gender is an especially important variable to consider in the context of generalization (e.g., Grace et al., 2013). 
Depressive Symptoms, Hopelessness, and MI incidence

\section{Funding Acknowledgments}

We thank the staff of the former Research Institute of Public Health, University of Kuopio, and Kuopio Research Institute of Exercise Medicine for the basic data collection in the KIHD study.

The KIHD study was supported by grants from the Academy of Finland (grants 132552, 118551, 41471, 1041086 and 2041022), the Ministry of Education of Finland (grants 167/722/96, 157/722/97, 156/722/98), and the National Heart, Lung and Blood Institute of the USA (grant HL44199). 
Depressive Symptoms, Hopelessness, and MI incidence

\section{References}

Abramson LY, Metalsky GI and Alloy LB (1989). Hopelessness depression: A theory-based subtype of depression. Psychological Review 96: 358-372.

Ai AL, Hopp F, Tice TN and Koenig H (2013). Existential relatedness in light of eudemonic well-being and religious coping among middle-aged and older cardiac patients. Journal of Health Psychology 18: 368-382.

American Heart Association (2013). AHA statistical update. Heart disease and stroke statistics2013 update. Retrieved on June 14, 2013 from http://circ.ahajournals.org/content/127/1/e6.full

American Psychiatric Association (2000). Diagnostic and Statistical Manual of Mental Disorders Fourth Edition Text Revision (DSM-IV-TR). Washington, DC: American Psychiatric Association.

Anda R, Williamson D, Jones D, Macera C, Eaker E, Glassman A and Marks J (1993). Depressed affect, hopelessness, and the risk of ischemic heart disease in a cohort of U.S. adults. Epidemiology 4: 285-294.

Beck AT (1976). Cognitive therapy and the emotional disorders. New York, NY: International University Press.

Bot M, Carney RM, Freedland KE, Rubin EH, Rich MW, Steinmeyer BC and Mann DL (2011). Inflammation and treatment response to sertraline in patients with coronary heart disease and comorbid major depression. Journal of Psychosomatic Research 71: 13-17.

Brown G and Harris T (1978). Social Origins of Depression. London, England: Tavistock.

Centers for Disease Control and Prevention (1998). Classification of diseases and injuries. Retrieved on July 18, 2011 from http://www.cdc.gov/nchs/icd/icd9.htm 
Cohen S, Kaplan GA and Salonen JT (1999). The role of psychological characteristics in the relation between socioeconomic status and perceived health. Journal of Applied Social Psychology 29: 445-468.

Cole DA and Maxwell SE (2003). Testing mediational models with longitudinal data: Questions and tips in the use of structural equation modeling. Journal of Abnormal Psychology 112: $558-577$.

Do DP, Down JB, Ranjit N, House JS and Kaplan GA (2010). Hopelessness, depression, and early markers of endothelial dysfunction in U.S. adults. Psychosomatic Medicine 72: 613-619.

Everson SA, Goldberg DE, Kaplan GA, Cohen RD, Pukkala E, Tuomilehto J and Salonen JT (1996). Hopelessness and risk of mortality and incidence of myocardial infarction and cancer. Psychosomatic Medicine 58: 113-121.

Everson SA, Kaplan GA, Goldberg DE and Salonen JT (2000). Hypertension incidence is predicted by high levels of hopelessness in Finnish men. Hypertension 35: 561-567.

Everson SA, Roberts RE, Goldberg DE and Kaplan GA (1998). Depressive symptoms and increased risk of stroke mortality over a 29-year period. Archives of Internal Medicine 158: $1133-1138$.

Fiedorowicz JGHJ and Merikangas KR (2011). The association between mood and anxiety disorders with vascular diseases and risk factors in a nationally representative sample. Journal of Psychosomatic Research 70: 145-154.

Fuller-Thomson E, Brennenstuhl S and Frank J (2010). The association between childhood physical abuse and heart disease in adulthood: Findings from a representative community sample. Child Abuse \& Neglect 34: 689-698. 
Glassman AH, Bigger JrT and Gaffney M (2009). Psychiatric characteristics associated with long-term mortality among 361 patients having an acute coronary syndrome and major depression. Seven-year follow-up of SADHART participants. Archives of General Psychiatry 66: 1022-1029.

Grace SL, Yee J, Reid RD and Stewart DE (2013). Measurement of depressive symptoms among cardiac patients: Should sex differences be considered? Journal of Health Psychology.

Grippo AJ and Johnson AK (2002). Biological mechanisms in the relationship between depression and heart disease. Neuroscience \& Biobehavioral Reviews 26: 941-962.

Haibe-Kains B, Desmedt C, Sotiriou C and Bontempi G (2008). A comparative study of survival models for breast cancer prognostication based on microarray data: Does a single gene beat them all? Bioinformatics 24: 2200-2208.

Kaplan GA, Roberts RE, Camacho TC and Coyne JC (1987). Psychosocial predictors of depression. American Journal of Epidemiology 125: 206-220.

Kuper H, Marmot M and Hemingway H (2002). Systematic review of prospective cohort studies of psychosocial factors in the etiology and prognosis of coronary heart disease. Seminars in Vascular Medicine 2: 267-314.

Leung YW, Flora DB, Gravely S, Irvine J, Carney RM and Grace SL (2012). The impact of premorbid and postmorbid depression onset on mortality and cardiac morbidity among patients with coronary heart disease: meta-analysis. Psychosomatic Medicine 74: 786801.

Lopez AD, Mathers CD, Ezzati M, Jamison DT and Murray CJL (2006). Global Burden of Disease and Risk Factors. Washington DC: World Bank \& Oxford University Press. 
Mathers CD and Loncar D (2006). Projections of global mortality and burden of disease from 2002 to 2030. PLoS Medicine 3: 2011 - 2030.

Nicholson A, Kuper H and Hemingway H (2006). Depression as an aetiologic and prognostic factor in coronary heart disease: a meta-analysis of 6362 events among 146,538 participants in 54 observational studies. European Heart Journal 27: 2763-2774.

Patten SB, Williams JVA, Lavorato DH, Modgill G, Jetté N and Eliasziw M (2008). Major depression as a risk factor for chronic disease incidence: longitudinal analyses in a general population cohort. General Hospital Psychiatry 30: 407-413.

Pizzi C, Rutjes AWS, Costa GM, Fontana F, Mezzetti A and Manzoli L (2011). Meta-analysis of Selective Serotonin Reuptake Inhibitors in patients with depression and coronary heart disease. American Journal of Cardiology 107: 972-979.

Pollitt RA, Daniel M and Kaufman JS (2005). Mediation and modification of the association between hopelessness, hostility, and progression of carotid atherosclerosis. Journal of Behavioral Medicine 28: 53-64.

Pössel P (2011). Can beck's theory of depression and the response style theory be integrated? Journal of Counseling Psychology 58: 618-629.

Pössel P and Hautzinger M (2006). Effekte pharmakologischer und psychotherapeutischer Interventionen auf Depressionen bei Kindern und Jugendlichen. [Effects of pharmacoand psychotherapeutic interventions of depression in children and adolescents.] Zeitschrift fuer Kinder- und Jugendpsychiatrie 34: 243-255.

Pössel, P. \& Knopf, K. (2011). Bridging the gaps: An attempt to integrate three major cognitive depression models. Cognitive Therapy and Research 35: 342-358. 
Roberts RE and O'Keefe SJ (1981). Sex differences in depression reexamined. Journal of Health and Social Behavior 22: 394-400.

Rosenbaum DL, White KS and Gervino EV (2012). The impact of perceived stress and perceived control on anxiety and mood disorders in noncardiac chest pain. Journal of Health Psychology 17: 1183-1192.

Salonen JT (1988). Is there a continuing need for longitudinal epidemiologic research? The kuopio ischemic heart disease risk factor study. Annals of Clinical Research 20: 46-50.

Sanjuan P, Arranz H and Castro A (2012). Pessimistic attributions and coping strategies as predictors of depressive symptoms in people with coronary heart disease. Journal of Health Psychology 17: 886-895.

Schmidt SD, Blank TO, Bellizzi KM and Park CL (2012). The relationship of coping strategies, social support, and attachment style with posttraumatic growth in cancer survivors. Journal of Health Psychology 17: 1033-1040.

Stamatakis KA, Lynch J, Everson SA, Raghunathan T, Salonen JT and Kaplan GA (2004). Self-esteeem and mortality: Prospective evidence from a population-based study. Annals of Epidemiology 14: 58-65.

Student (1908). The Probable Error of a Mean. Biometrika 6: 1-25.

Tolmunen T, Hintikka J, Voutilainen S, Ruusunen A, Alfthan G, Nyyssönen K, Viinamäki H, Kaplan GA and Salonen JT (2004). Association between depressive symptoms and serum concentrations of homocysteine in men: A population study. The American Journal of Clinical Nutrition 80: 1574-1578.

Tully PJ, Winefield HR, Baker RA, Turnbull DA and de Jonge P (2011). Confirmatory factor analysis of the Beck Depression Inventory-II and the association with cardiac morbidity 
Depressive Symptoms, Hopelessness, and MI incidence

and mortality after coronary revascularization. Journal of Health Psychology 16: 584595.

Valtonen M, Laaksonen DE, Tolmunen T, Nyyssönen K, Viinamäki H, Kauhanen J and Niskanen L (2008). Hopelessness - novel facet of the metabolic syndrome in men. Scandinavian Journal of Public Health 36: 795-802.

Van der Kooy K, van Hout H, Marwijk H, Marten H, Stehouwer C and Beekman A (2007). Depression and the risk for cardiovascular diseases: systematic review and meta analysis. International Journal of Geriatric Psychiatry 22: 613-626.

Whipple MO, Lewis TT, Sutton-Tyrrell K, Matthews KA, Barinas-Mitchell E, Powell LH and Everson-Rose SA (2009). Hopelessness, depressive symptoms, and carotid atherosclerosis in women: The study of women's health across the nation (SWAN) heart study. Stroke 40: $3166-3172$.

World Health Organization (2007). International statistical classification of diseases and related health problems. Retrieved on July 18, 2011 from http://apps.who.int/classifications/apps/icd/icd10online/ 
Table 1

Cox proportional hazards regression models predicting myocardial infarctions from scores on depression and hopelessness scales

\begin{tabular}{|c|c|c|c|c|c|c|c|c|}
\hline & & & \multicolumn{2}{|c|}{ Depressive symptoms - MI } & \multicolumn{2}{|c|}{ Hopelessness - MI } & \multicolumn{2}{|c|}{$\begin{array}{l}\text { Hopelessness \& Depressive } \\
\text { symptoms - MI }\end{array}$} \\
\hline & $M$ & $\mathrm{SD}$ & $\mathrm{B}(S E)$ & HR $(95 \%$ CI $)$ & $\mathrm{B}(S E)$ & HR $(95 \%$ CI $)$ & $\mathrm{B}(S E)$ & HR (95\% CI) \\
\hline Depressive & 1.59 & 1.91 & $0.06(0.03)$ & $1.06(1.00-1.13)^{*}$ & --- & ----- & $0.04(0.03)$ & $1.04(0.98-1.11)$ \\
\hline \multicolumn{9}{|l|}{ symptoms } \\
\hline Hopelessness & 2.45 & 1.93 & --- & ----- & $0.08(0.03)$ & $1.08(1.02-1.15)^{*}$ & $0.07(0.03)$ & $1.07(1.00-1.14)^{*}$ \\
\hline \multicolumn{9}{|l|}{ Covariates } \\
\hline Age & 51.92 & 5.80 & $0.05(0.01)$ & $1.05(1.03-1.08)^{* * *}$ & $0.04(0.01)$ & $1.04(1.02-1.07)^{* * *}$ & $0.04(0.01)$ & $1.04(1.02-1.07)^{* * *}$ \\
\hline Resting SBP & 133.30 & 16.35 & $0.01(0.00)$ & $1.01(1.01-1.02)^{* * *}$ & $0.01(0.00)$ & $1.01(1.01-1.02)^{* * *}$ & $0.01(0.00)$ & $1.01(1.01-1.02)^{* * *}$ \\
\hline LDL & 3.93 & 0.96 & $0.34(0.06)$ & $1.40(1.24-1.57)^{* * *}$ & $0.34(0.06)$ & $1.40(1.24-1.58)^{* * *}$ & $0.34(0.06)$ & $1.40(1.24-1.58)^{* * *}$ \\
\hline HDL & 1.30 & 0.29 & $0.54(0.22)$ & $0.58(0.38-0.90)^{*}$ & $-0.45(0.23)$ & $0.64(0.41-9.90)^{*}$ & $-0.46(0.23)$ & $0.63(0.41-0.98)^{*}$ \\
\hline Waist-hip ratio & 0.94 & 0.06 & $2.22(0.89)$ & $9.21(1.62-52.43)^{*}$ & $2.60(0.98)$ & $13.39(1.98-90.58)^{* *}$ & $2.54(0.98)$ & $12.63(1.86-85.77)^{* *}$ \\
\hline
\end{tabular}

Note. $\mathrm{SBP}=$ systolic blood pressure; $\mathrm{LDL}=$ low-density lipoprotein; $\mathrm{HDL}=$ high-density lipoprotein; Org. = Organizational

$* p<.05 ; * * p<.01 ; * * * p<.001$ 